\title{
Investigating aberrantly expressed microRNAs in peripheral blood mononuclear cells from patients with treatment-resistant schizophrenia using miRNA sequencing and integrated bioinformatics
}

\author{
XU YOU ${ }^{1 *}$, YUNQIAO ZHANG ${ }^{1 *}$, QING LONG ${ }^{1 *}$, ZIJUN LIU $^{1}$, XIAO MA ${ }^{1}$, ZIXIANG LU ${ }^{2}$, \\ WEI YANG ${ }^{3}$, ZIQIAO FENG ${ }^{1}$, WENGYU ZHANG ${ }^{1}$, ZHAOWEI TENG $^{1}$ and YONG ZENG ${ }^{1}$ \\ ${ }^{1}$ Research Management Department, The Sixth Affiliated Hospital, Kunming Medical University, \\ Yuxi, Yunnan 653100; ${ }^{2}$ Psychiatric Ward, Honghe Second People's Hospital, Honghe, Yunnan 654399; \\ ${ }^{3}$ Psychiatric Ward, Yuxi Second People's Hospital, Yuxi, Yunnan 653100, P.R. China
}

Received March 31, 2020; Accepted July 31, 2020

DOI: $10.3892 / \mathrm{mmr} .2020 .11513$

\begin{abstract}
Treatment-resistant schizophrenia (TRS) is a common phenotype of schizophrenia that places a considerable burden on patients as well as on society. TRS is known for its tendency to relapse and uncontrollable nature, with a poor response to antipsychotics other than clozapine. Therefore, it is urgent to identify objective biological markers, so as to guide its treatment and associated clinical work. In the present study, the peripheral blood mononuclear cells (PBMCs) of patients with TRS and a healthy control group, which were gender-, age- and ethnicity-matched, were subjected to microRNA (miRNA/miR) sequencing to screen out the top three miRNAs with the highest fold change values. These were then validated in the TRS $(n=34)$ and healthy control $(n=31)$ groups by reverse transcription-quantitative PCR. For two of the top three miRNAs, the PCR results were in accordance with the sequencing result $(\mathrm{P}<0.01)$, while the third miRNA exhibited the opposite trend $(\mathrm{P}<0.01)$. To elucidate the functions of these two miRNAs, Homo sapiens (hsa)-miR-218-5p and hsa-miR-1262 and their regulatory network, target gene prediction was first performed using online TargetScan and Diana-micro T software. Bioinformatics analysis was then performed using functional enrichment analysis to determine the Gene
\end{abstract}

Correspondence to: Dr Yong Zeng or Dr Zhaowei Teng, Research Management Department, The Sixth Affiliated Hospital, Kunming Medical University, 21 Nieer Road, Yuxi, Yunnan 653100, P.R. China E-mail: zengyong1388851037@163.com

E-mail: tengzhaowei2002@163.com

${ }^{*}$ Contributed equally

Key words: treatment-resistant schizophrenia, microRNA, biomarker, diagnosis
Ontology terms in the category biological process and the Kyoto Encyclopedia of Genes and Genomes pathways. It was revealed that these target genes were markedly associated with the nervous system and brain function, and it was obvious that the differentially expressed miRNAs most likely participated in the pathogenesis of TRS. A receiver operating characteristic curve was generated to confirm the distinct diagnostic value of these two miRNAs. It was concluded that aberrantly expressed miRNAs in PMBCs may be implicated in the pathogenesis of TRS and may serve as specific peripheral blood-based biomarkers for the early diagnosis of TRS.

\section{Introduction}

Schizophrenia (SCZ) is a complex and severe psychiatric disorder that affects $\sim 1 \%$ of the general population worldwide, placing a heavy burden on the patients, the patients' family and society (1). Although administration of antipsychotics remains the major therapeutic strategy for SCZ, one-third of patients are insensitive to them (2). Patients who only have a minor or no response to at least two non-clozapine drugs at adequate doses and treatment cycles are diagnosed with treatment-resistant SCZ (TRS) according to the guidelines of the American Psychiatric Association (3). At present, frequent rehospitalization and repeated episodes of symptoms in patients with TRS increase the burden on patients and their families (4). It is currently thought that a combination of environmental and genetic factors is most likely to be the major cause of SCZ. However, the precise diagnosis of SCZ still only depends on clinical symptoms, without the help of sensitive and objective molecular markers. It is therefore urgent and necessary to identify sensitive biomarkers for the early and accurate diagnosis of SCZ, so that earlier and effective therapeutic intervention may be provided (5-7). Nowadays, strong evidence from psychiatric clinical studies has indicated that environmental factors may result in aberrant gene expression in the central nervous system (CNS), which may be the 
major cause of symptom onset $(8,9)$. A study has indicated that $\geq 50 \%$ of changes in gene expression in the CNS are involved in the changes in the expression of the same genes in peripheral tissues (10). Since brain tissues, several of which have deeply located and complex structures with difficult access, are challenging to directly investigate, the present study attempted to examine and elucidate potential biomarkers for SCZ via a number of blood-derived gene expression analyses (10-14). By using this non-invasive method, effective peripheral blood biomarkers may be discovered that may be considerably helpful in promoting earlier TRS diagnosis, as well as enabling the concise assessment of treatment response and even precisely predicting prognosis.

Recently, increasing attention has been paid to the regulatory mechanisms of microRNAs (miRNAs/miRs) (15-17), which are considered to be in charge of gene expression monitoring through the inhibition of mRNA translation (18). Previous studies have indicated that changes in the expression of certain mRNAs reflect parallel changes in the CNS (19) and peripheral blood $(20,21)$. The notion that certain miRNAs may be sensitive biomarkers for SCZ has gained much research interest (22-27), since alterations in their expression in peripheral blood mononuclear cells (PBMCs) may provide valuable clues for the earlier and more precise diagnosis and more effective treatment of SCZ (28). It is worth noting that abnormally expressed miRNAs have been detected in the PBMCs and plasma of patients with SCZ. In a recent study, Lai et al (28) indicated that 7 miRNAs, namely miR-31, miR-431, miR-433, miR-107, miR-134, miR-99b and miR-487b, were downregulated in the PBMCs derived from 112 patients with mental illness, as compared with those of a control group of 76 non-psychotic patients. Of note, the imprinted delta-like homologue 1 and the type III iodothyronine deiodinase (DLK1-DIO3) region of 14q32 from PBMCs was considered an SCZ-associated miRNA signature (28). In that study, a total of 7 differentially expressed miRNAs [Homo sapiens (hsa)-miR-34a, miR-449a, miR-564, miR-432, miR-548d, miR-572 and miR-652] were identified to be closely associated with emotional retardation, restricted language and other neurocognitive dysfunctions in 30 patients with SCZ, as compared with 30 controls (28). In another noteworthy study, specific miRNA30e in the plasma was identified as a biomarker of SCZ diagnosis from the expressed profiles of 9 SCZ-associated miRNAs in PBMCs by means of reverse transcription-quantitative PCR (RT-qPCR) (29). Subsequently, miR-130b and miR-193a-3p were identified as biomarkers for the diagnosis of SCZ by high-throughput sequencing and RT-qPCR (30). The early growth response protein-1 (EGR1)/miR-30a-5p/neurogenic differentiation 1 (NEUROD1) axis was proposed as a promising biomarker for the diagnosis and treatment monitoring of patients with SCZ with acute mental states based on RT-qPCR technology (31).

miRNA analysis may therefore be used to effectively elucidate the etiology of SCZ and identify potential differences among mental illnesses with similar but unclear clinical symptoms (32). The present study aimed to identify novel and specific biomarkers to earlier and more precisely diagnose patients with TRS and implement effective therapeutic strategies. Second-generation high-throughput sequencing technology and bioinformatics were used to screen out and analyze the top three abnormally expressed miRNAs in PBMCs from 6 patients with TRS and 6 healthy controls. Next, RT-qPCR was used to validate the significantly differentially expressed miRNAs in a larger sample of cases and healthy controls. The newly identified target genes of the two confirmed differentially expressed miRNAs were predicted with the online software TargetScan and Diana-micro T, and bioinformatics methods further indicated that these target genes were closely associated with functions of the nervous system. Finally, these two miRNAs were proposed as diagnostic biomarkers for TRS, thereby providing a novel, simple and convenient diagnostic tool for this condition.

\section{Materials and methods}

Screening and collection of cases. The subjects of the present study were divided into two groups, the case group and the control group. For the case group, a total of 34 patients with TRS (17 males and 17 females) were recruited from the Psychiatric inpatient ward of Honghe Second People's Hospital and Yuxi Second People's Hospital (Yunnan, China). The inclusion criteria were as follows: i) All patients met the mental illness criteria outlined in the Diagnostic and Statistical Manual Fifth Edition Criteria for SCZ (DSM-5; American Psychiatric Association; 2013) (33); ii) Complete clinical data and iii) Voluntary participation in the study. These 34 patients were screened by 2 independent experienced deputy chief physicians, according to the inclusion criteria. The exclusion criteria were conditions such as physical or cerebral organic disease, other types of mental illness, drug abuse prior to hospitalization, patients who received modified electroconvulsive therapy within 3 months from enrollment, and patients with a history of blood transfusion within the last month.

The control group included 31 healthy individuals with no family history of major mental illness recruited at the Physical Examination Center of the Sixth Affiliated Hospital of Kunming Medical University (Kunming, China). Of the 31 control individuals, 16 were males and 15 females, all of whom had a normal physical examination and psychological examination to exclude the presence of SCZ, none of whom had suffered any recent adverse life events. The age and sex ratio of patients and controls was 2:1.

All included subjects were older than 18 years. According to the requirements of the ethics committee, written informed consent for the case group was required not only from the patient but also one family member/guardian, while for the healthy control group, informed consent was provided by each subject themselves. The present study was approved by the ethics committees of Honghe Second People's Hospital (Honghe, Yunnan; approval no. 20190013) and The Sixth Affiliated Hospital of Kunming Medical University (Yuxi, Yunnan; approval no. 2020kmykdx6h58).

Extraction of PBMCs. Approximately $5 \mathrm{ml}$ peripheral venous blood was collected by quick blood sampling from 65 cases and processed within $1 \mathrm{~h}$. This procedure was carried out according to the manufacturer's protocol (Dakwei Biological 
Engineering). In brief, fresh whole blood was collected in EDTA-K2 anti-coagulation tubes and then diluted with an equal volume of isotonic solution (normal saline). Subsequently, $15 \mathrm{ml}$ separation liquid was added to the centrifuge tube. The volume ratio of the separation liquid, anticoagulated undiluted whole blood and isotonic solution (physiological saline) was $1: 1: 1$. Next, the sample was centrifuged at 700-800 x $\mathrm{g}$ for 20-30 min at room temperature. Between the plasma and separation layers was a thin and dense white film. The white film layer was carefully pipetted into another centrifuge tube. The horizontal rotor was then centrifuged at $250 \mathrm{x} g$ at room temperature for $10 \mathrm{~min}$ and the supernatant was discarded. Washing was repeated 1-2 times. Finally, $1 \mathrm{ml}$ TRIzol ${ }^{\circledR}$ (Invitrogen; Thermo Fisher Scientific, Inc.) was added and the sample was mixed. A total of $1.5 \mathrm{ml}$ was added to the labeled cryotube. The cryotube was tightly sealed with parafilm and stored at $-80^{\circ} \mathrm{C}$.

RNA extraction and miRNA sequencing. RNA samples were harvested from 6 patients with TRS ( 3 males, 3 females) and 6 healthy controls ( 3 males, 3 females). The patients in the experimental groups were age-matched. Samples were delivered to Guangzhou Gidio Biotechnology Co., Ltd. for the extraction of small RNA and the construction of small RNA libraries. In brief, RNA was extracted using the TRIzol method, followed by detection of RNA purity with a NanoDrop 2000 (Thermo Fisher Scientific, Inc.) and RNA integrity with an Agilent 2100 Bioanalyzer (Agilent Technologies GmbH). Small RNAs were obtained by gel electrophoresis from total RNA, and 5' and 3' ends were joined. RT-qPCR was performed to construct small RNA libraries. Subsequently, the constructed libraries were tested for quality and yield using an Agilent 2100 and ABI StepOnePlus Real-Time PCR System (Thermo Fisher Scientific, Inc.). An Illumina HiSeq2000 (Illumina, Inc.) was used for sequencing. All procedures were in strict accordance with the manufacturer's protocols (Gidio Biotechnology Co., Ltd.)

$R T$ - $q P C R$. RT-qPCR was used to validate the top three aberrantly expressed miRNAs in 34 patients with TRS and 31 healthy controls. In brief, after total RNA was isolated from PBMCs, complementary (c)DNA was synthetized using the miRcute Plus miRNA First-Strand cDNA kit and miRcute Plus miRNA qPCR kit (SYBR Green) according to the manufacturer's protocol. RT-qPCR was performed in the Quantitative PCR Reaction Amplifier (CFX96 Touch; Bio-Rad Laboratories, Inc.), with the reaction system including 2X miRcute Plus miRNA Premix (SYBR and ROX), PCR forward primer and PCR reverse primer. The primers for three miRNAs are summarized in Table SI. Detailed experimental instruments and reagents are included in Tables SII and SIII. $\mathrm{h}$-actin for miRNA was applied to normalize the results. The expression levels of miRNAs were calculated using the $2^{-\Delta \Delta \mathrm{Cq}}$ method (34). All RT-qPCR validation procedures were performed by Wuhan Genecreate Biological Engineering Co., Ltd.

miRNA target gene prediction and bioinformatics analysis. TargetScan release 7.2 (http://www.targetscan.org/) (35) and Diana-microT v5.0 (http://diana.imis.athena-innovation.
Table I. Demographic data of the cases and healthy controls.

\begin{tabular}{lccc}
\hline Item & Cases $(\mathrm{n}=34)$ & Controls $(\mathrm{n}=31)$ & P-value $^{\mathrm{a}}$ \\
\hline Sex & & & 0.897 \\
Male & $17(50.0)$ & $16(51.6)$ & \\
Female & $17(50.0)$ & $15(48.4)$ & \\
Age (years) & $41.82 \pm 11.414$ & $37.06 \pm 11.363$ & 0.956 \\
\hline
\end{tabular}

Values are expressed as $\mathrm{n}(\%)$ or the mean \pm standard deviation. ${ }^{\mathrm{a}} \mathrm{A}$ t-test was performed for quantitative variables and a $\chi^{2}$ test was performed for categorical variables.

gr/DianaTools/index.php?r=microT_CDS/index) (36), which are two major and widely accepted open databases, were used to predict the target genes of the significantly differentially expressed miRNAs. Subsequently, the target genes obtained from the two databases were intersected and underwent further GO and Kyoto Encyclopedia of Genes and Genomes (KEGG) pathway enrichment analysis. GO annotation and KEGG enrichment analysis of the target genes were performed using the OmicShare tools (37), a free online platform for data analysis (http://www.omicshare.com/tools). The GO project covers three classical domains, namely biological process (BP), cellular component and molecular function. The KEGG is a database that integrates genome, chemistry, and system function information, and aims to reveal the genetic and chemical blueprint of biological phenomena. $\mathrm{P}<0.05$ was considered to indicate a statistically significant result. The data were analyzed and obtained using the OmicShare cloud platform (https://www.omicshare. com/).

Statistical analysis. SPSS 22 (IBM Corp.) and GraphPad Prism 7.0 (GraphPad Software, Inc.) were used for data analysis. The $\chi^{2}$ test for the qualitative variables and the $\mathrm{t}$-test for the quantitative variables were respectively used for the demographic analysis of the case and control groups. The Mann-Whitney U test was used to compare the differential expression levels of miRNAs. Receiver operating characteristic (ROC) curve analysis was used to assess the area under the curve (AUC). $\mathrm{P}<0.05$ was considered to indicate a statistically significant difference.

\section{Results}

Demographic data of the subjects. All subjects were Han Chinese. The mean age (mean \pm standard deviation) of TRS cases and controls was $41.82 \pm 11.414$ and $37.06 \pm 11.363$ years, respectively. There were no significant differences in terms of age or sex between the cases and healthy controls (Table I).

miRNA expression profile analysis. Analysis of the miRNA sequencing data revealed that a total of 34 miRNAs were significantly differentially expressed in the 6 cases of TRS as compared with the 6 controls ( $\mid \log 2$ (fold change) $\mid>1$; $\mathrm{P}<0.05)$. Among the six miRNAs with significantly differential expression, two were markedly downregulated and four 
Table II. Differentially expressed miRNAs in peripheral blood mononuclear cells from cases vs. healthy controls.

\begin{tabular}{|c|c|c|c|}
\hline miRNA & $\log 2 \mathrm{FC}$ & P-value & Direction of change \\
\hline hsa-miR-1185-1-3p & 1.38018786 & 0.014888805 & Up \\
\hline hsa-miR-1185-5p & 2.055813817 & 0.01030376 & Up \\
\hline hsa-miR-1197 & 1.669823494 & 0.003827819 & Up \\
\hline hsa-miR-1262 & 4.449671416 & 0.01862468 & Up \\
\hline hsa-miR-127-5p & 1.663957827 & 0.010180048 & Up \\
\hline hsa-miR-1304-3p & -7.019942788 & 0.004047489 & Down \\
\hline hsa-miR-136-3p & 1.536408035 & 0.010754284 & Up \\
\hline hsa-miR-154-3p & 2.635532322 & 0.001485503 & Up \\
\hline hsa-miR-181b-3p & 2.399752284 & 0.037210273 & Up \\
\hline hsa-miR-218-5p & 4.289711531 & 0.046989353 & Up \\
\hline hsa-miR-3653-3p & -3.106775941 & 0.031498569 & Down \\
\hline hsa-miR-369-3p & 1.219176104 & 0.035538469 & Up \\
\hline hsa-miR-370-3p & 1.383232956 & 0.034880938 & Up \\
\hline hsa-miR-376a-3p & 1.265825554 & 0.041422801 & Up \\
\hline hsa-miR-376c-3p & 1.281059616 & 0.019343549 & Up \\
\hline hsa-miR-377-3p & 1.68135408 & 0.004843865 & Up \\
\hline hsa-miR-379-3p & 1.33354957 & 0.02582764 & Up \\
\hline hsa-miR-382-5p & 1.164588341 & 0.042177088 & Up \\
\hline hsa-miR-409-3p & 1.215682697 & 0.031732848 & Up \\
\hline hsa-miR-409-5p & 1.33800193 & 0.02587475 & Up \\
\hline hsa-miR-410-3p & 1.144646395 & 0.04797714 & Up \\
\hline hsa-miR-411-3p & 1.188991694 & 0.049323611 & Up \\
\hline hsa-miR-431-3p & 1.433665135 & 0.037350442 & Up \\
\hline hsa-miR-431-5p & 1.703040789 & 0.003189292 & Up \\
\hline hsa-miR-432-5p & 1.265157654 & 0.035781474 & Up \\
\hline hsa-miR-485-3p & 1.118394154 & 0.046032191 & Up \\
\hline hsa-miR-490-3p & 1.792169025 & 0.040858315 & Up \\
\hline hsa-miR-493-5p & 1.499534768 & 0.008400669 & Up \\
\hline hsa-miR-539-3p & 1.558249279 & 0.010618161 & Up \\
\hline hsa-miR-543 & 1.112920365 & 0.044806101 & Up \\
\hline hsa-miR-654-5p & 1.433656533 & 0.014340331 & Up \\
\hline hsa-miR-655-3p & 1.943235022 & 0.013931082 & Up \\
\hline hsa-miR-6810-3p & 2.183414515 & 0.042813132 & Up \\
\hline hsa-miR-758-3p & 1.339956277 & 0.01416857 & Up \\
\hline
\end{tabular}

FC, fold change; miRNA/miR, microRNA; hsa, Homo sapiens.

were upregulated (Table II). A heat map was constructed to display the differential hierarchical clustering distribution between TRS and control samples (Fig. 1).

$R T-q P C R$ validation. The three miRNAs (hsa-miR-1262, hsa-miR-1304-3p and hsa-miR-218-5p) with the highest $\log _{2} \mathrm{FC}$ values $\left(\log _{2} \mathrm{FCl}>4\right)$ were verified in 34 TRS cases and 31 controls by RT-qPCR. Of these three miRNAs, hsa-miR-218-5p and hsa-miR-1262 were markedly upregulated according to the next-generation sequencing results, while hsa-miR-1304-3p was markedly downregulated. The RT-qPCR results indicated that the expression of hsa-miR-218-5p and hsa-miR-1262 in the TRS cases was markedly higher than that in the controls, which was consistent with the sequencing results $(\mathrm{P}<0.01)$. However, the expression of hsa-miR-1304-3p in the TRS cases was also markedly higher than that in the controls, which was inconsistent with the sequencing results $(\mathrm{P}<0.01$; Fig. 2$)$.

Prediction of miRNA target genes. By using two publicly available and widely accepted miRNA target gene prediction databases, TargetScan and Diana-micro T, the target genes of hsa-miR-218-5p and hsa-miR-1262 were predicted (Fig. 3A and B, respectively). For hsa-miR-218-5p, 187 target genes were predicted, while for hsa-miR-1262 had only 51 target genes were obtained. Next, a miRNA-gene co-expression network was generated to summarize the interaction between miRNAs and target genes (Fig. 4). 


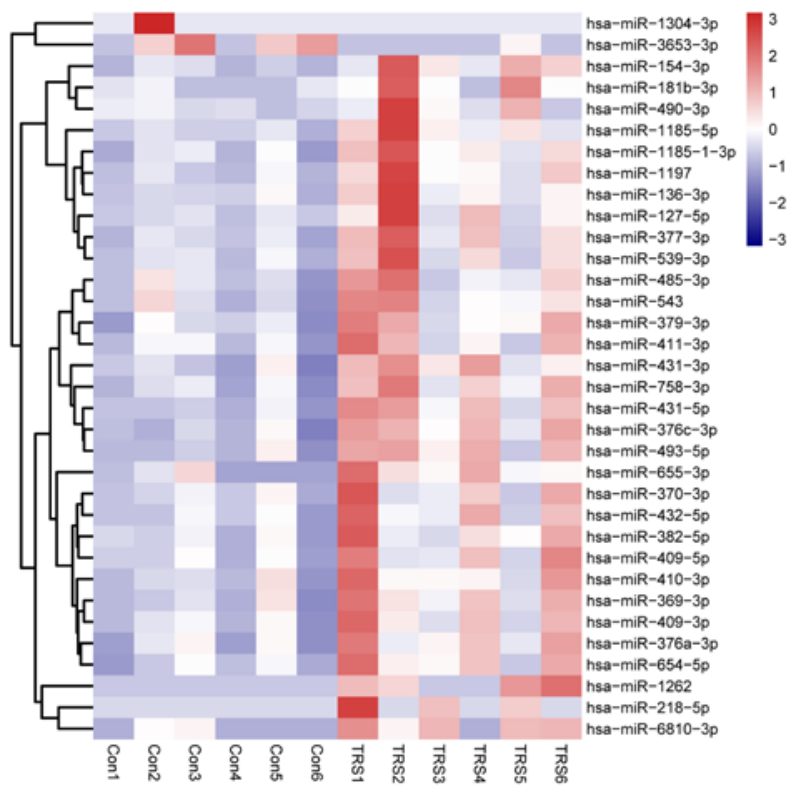

Figure 1. Heat map of 34 differentially expressed miRNAs between 6 cases of TRS and 6 controls. The columns display miRNA species and the rows individual samples. The color labels of each cell represent the expression level of miRNAs. Red represents upregulation and blue downregulation. Con, control; TRS, treatment-resistant schizophrenia; miRNA/miR, microRNA; hsa, Homo sapiens.

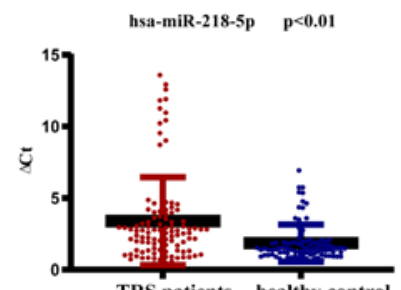

TRS patients healthy control

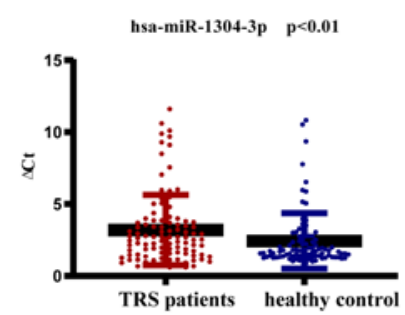

Figure 2. Reverse transcription-quantitative PCR was performed to verify the expression of miRNAs from peripheral blood mononuclear cells in a large sample (TRS, $n=34$; control, $n=31$ ). miRNA levels are normalized to $\mathrm{H}$-actin. GraphPad Prism 7 was used to plot the data and the Mann-Whitney U-test was performed and analyze significant differences. Data are presented as the mean \pm SD. TRS, treatment-resistant schizophrenia; miRNA/miR, microRNA; hsa, Homo sapiens.

GO and pathway enrichment analysis. Target genes of these two miRNAs were enriched by GO annotation and KEGG analysis. The results for the top $20 \mathrm{GO}$ terms enriched by the target genes of hsa-miR-218-5p are presented in Fig. 5A. In the GO category BP, the target genes of hsa-miR-218-5p were prominently involved in various physiological and pathophysiological processes, including nervous system development, generation of neurons, cell morphogenesis regarding neuron differentiation, synapse organization, neuron projection
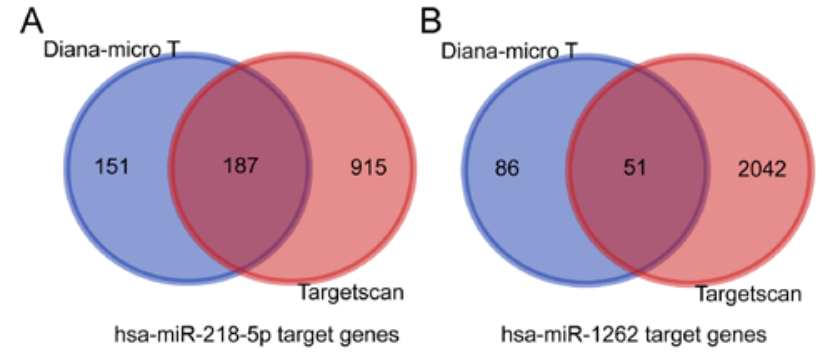

Figure 3. Venn diagram of genes from three miRNA target prediction datasets. Target genes of (A) hsa-miR-218-5p and (B) hsa-miR-1262. miRNA/miR, microRNA; hsa, Homo sapiens.

morphogenesis and neuron differentiation. BP terms associated with brain development and function are presented in Table III. KEGG pathway analysis for the target genes of hsa-miR-218-5p indicated a significant enrichment in several important pathways including the PI3K/Akt, dopaminergic synapse and AMPK signaling pathways, as well as the Hippo signaling pathway-multiple species (Fig. 5B).

In the category $\mathrm{BP}$, the $\mathrm{GO}$ analysis for the target genes of hsa-miR-1262 revealed their close association with BP regulation, biological regulation, cellular process regulation and cellular protein modification process (Fig. 5C). BP terms closely associated with brain development and function are presented in Table IV. KEGG pathway analysis for the target genes of hsa-miR-1262 indicated an obvious enrichment in crucial pathways, such as nonhomologous end-joining dopaminergic synapse oocyte meiosis and adrenergic signaling in cardiomyocytes (Fig. 5D).

Diagnostic evaluation of hsa-miR-218-5p and hsa-miR-1262. ROC curve analysis was performed to demonstrate the potential of the expression levels of the 2 miRNAs confirmed by RT-qPCR as biomarkers for distinguishing patients with TRS from healthy controls. As displayed in Fig. 6, the AUC for hsa-miR-218-5p was 0.80 (95\% CI, 0.7301-0.8604; $\mathrm{P}<0.01$; Fig. 6A), the best cutoff value was 0.62 (specificity, 0.826; sensitivity, 0.794).The AUC for hsa-miR-1262 was 0.69 (95\% CI, 0.6129-0.7669; P<0.01; Fig. 6B), the best cutoff value was 0.47 (specificity, 0.828; sensitivity, 0.637). The AUC for the combination of the 2 miRNAs was able to clearly distinguish patients with TRS from healthy controls with an AUC of 0.7501 (95\% CI, 0.7005-0.7998; P<0.01; Fig. 6C), the best cutoff value was 0.51 (specificity, 0.801 ; sensitivity, 0.711 ).

\section{Discussion}

TRS accounts for $30 \%$ of all cases of SCZ (38). Due to its refractory and complex nature, TRS is a unique and severe phenotype of SCZ and places a burden on the patients' family and society (39). Therefore, precision medicine for this particular type of SCZ requires to be urgently implemented in the clinic. Although precision medicine is a novel healthcare discipline (40) that gives priority to the unique characteristics of specific patients (41), its use in psychiatry is still lagging compared with other medical fields, since it relies on neuroimaging and/or biological technology to provide a benefit $(42,43)$. Several studies have made outstanding contributions to 


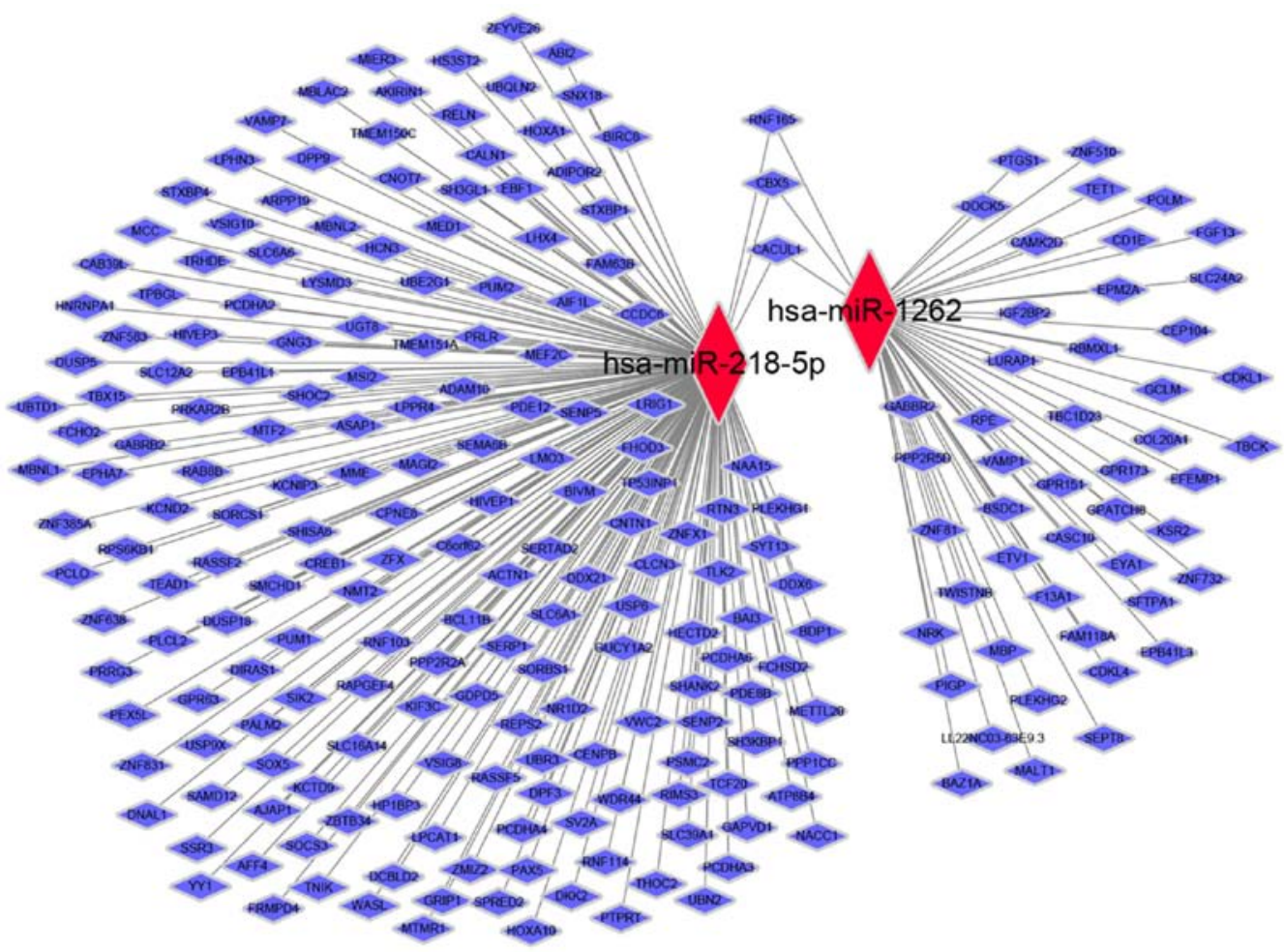

Figure 4. miRNA-gene co-expression network. Red represents the miRNA and blue represents the targets. miRNA/miR, microRNA; hsa, Homo sapiens.
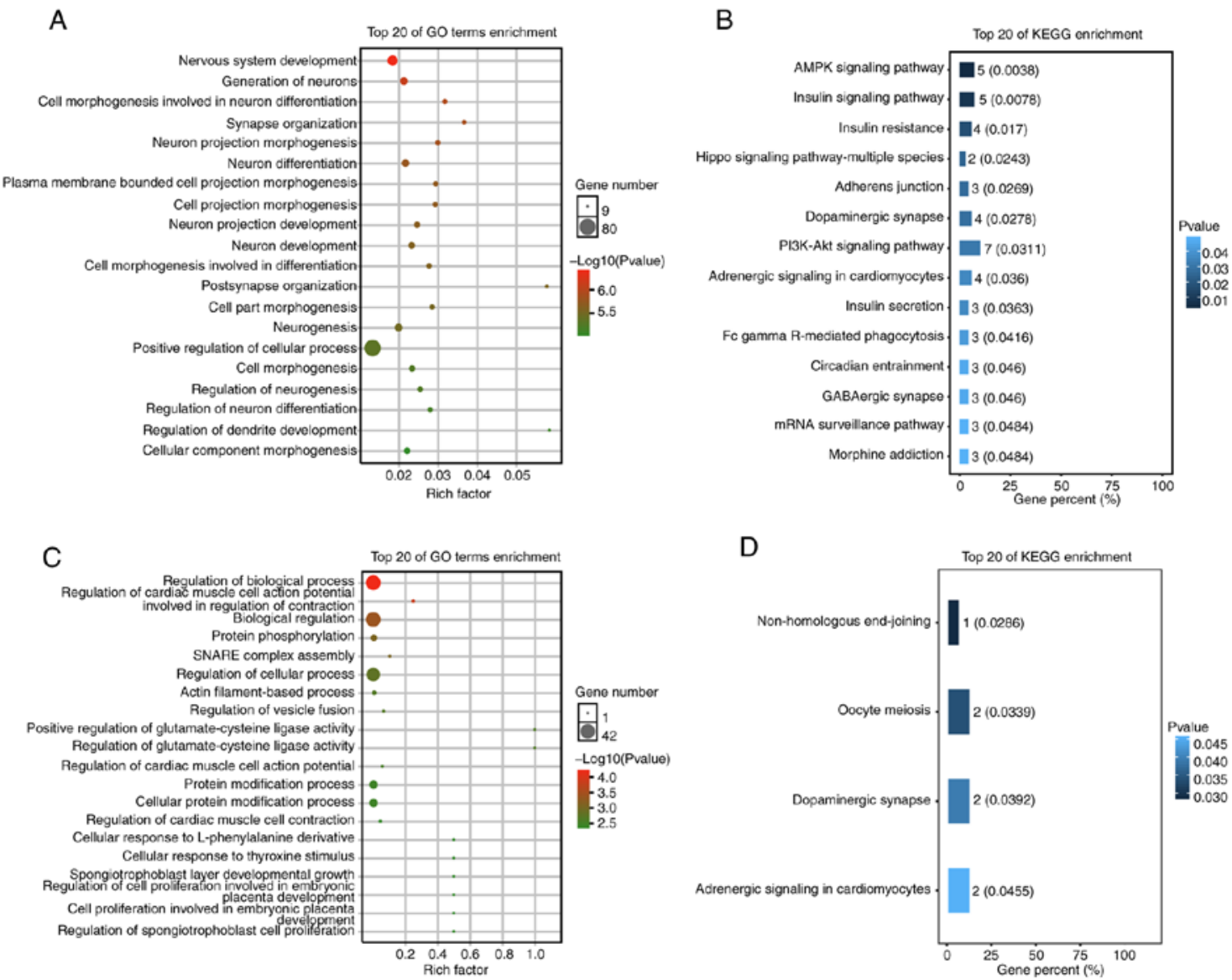

Figure 5. GO annotation and KEGG analysis of target genes of hsa-miR-218-5p and hsa-miR-1262. (A) Top 20 GO terms in the categories BB, CC and MF of the target genes of hsa-miR-218-5p ( $\mathrm{P}<0.05)$. (B) A total of 14 statistically significantly enriched KEGG pathways were identified ( $\mathrm{P}<0.05)$. (C) Top $20 \mathrm{GO}$ terms $(\mathrm{BB}, \mathrm{CC}$ and $\mathrm{MF})$ of the target genes of hsa-miR-1262 (P<0.05). (D) A total of four statistically significantly enriched KEGG pathways are presented $(\mathrm{P}<0.05)$. GO, Gene Ontology; KEGG, Kyoto Encyclopedia of Genes and Genomes; BP, Biological Process; CC, Cellular Component; MF, Molecular Function; miRNA/miR, microRNA; hsa, Homo sapiens. 
Table III. Top 20 enriched GO Biological Process terms associated with brain development and functions of hsa-miR-218-5p target genes.

\begin{tabular}{|c|c|c|c|}
\hline GO ID & GO term & Number of enriched genes & P-value \\
\hline GO:0007399 & Nervous system development & 46 & $3.79 \times 10^{-7}$ \\
\hline GO:0048699 & Generation of neurons & 34 & $7.75 \times 10^{-7}$ \\
\hline GO:0048667 & Cell morphogenesis involved in neuron differentiation & 19 & $1.15 \times 10^{-6}$ \\
\hline GO:0050808 & Synapse organization & 16 & $1.34 \times 10^{-6}$ \\
\hline GO:0048812 & Neuron projection morphogenesis & 20 & $1.40 \times 10^{-6}$ \\
\hline GO:0030182 & Neuron differentiation & 31 & $1.73 \times 10^{-6}$ \\
\hline GO:0031175 & Neuron projection development & 25 & $2.26 \times 10^{-6}$ \\
\hline GO:0048666 & Neuron development & 27 & $2.52 \times 10^{-6}$ \\
\hline GO:0099173 & Postsynapse organization & 10 & $2.78 \times 10^{-6}$ \\
\hline GO:0022008 & Neurogenesis & 34 & $3.36 \times 10^{-6}$ \\
\hline GO:0050767 & Regulation of neurogenesis & 22 & $5.84 \times 10^{-6}$ \\
\hline GO:0045664 & Regulation of neuron differentiation & 19 & $7.09 \times 10^{-6}$ \\
\hline GO:0050773 & Regulation of dendrite development & 9 & $8.09 \times 10^{-6}$ \\
\hline GO:0048523 & Negative regulation of cellular process & 72 & $1.06 \times 10^{-5}$ \\
\hline GO:0016358 & Dendrite development & 11 & $1.08 \times 10^{-5}$ \\
\hline GO:0060996 & Dendritic spine development & 7 & $2.48 \times 10^{-5}$ \\
\hline GO:0050807 & Regulation of synapse organization & 10 & $4.98 \times 10^{-5}$ \\
\hline GO:0060998 & Regulation of dendritic spine development & 6 & $5.14 \times 10^{-5}$ \\
\hline GO:0099504 & Synaptic vesicle cycle & 9 & $6.40 \times 10^{-5}$ \\
\hline GO:0050803 & Regulation of synapse structure or activity & 10 & $6.76 \times 10^{-5}$ \\
\hline
\end{tabular}

GO, gene ontology; miR, microRNA; hsa, Homo sapiens.

Table IV. Top 20 enriched GO Biological Process terms associated with brain development and functions of hsa-miR-1262 target genes.

\begin{tabular}{llrr}
\hline GO ID & \multicolumn{1}{c}{ GO term } & Number of enriched genes & P-value \\
\hline GO:0045196 & Establishment or maintenance of neuroblast polarity & 1 & 0.006 \\
GO:0045200 & Establishment of neuroblast polarity & 1 & 0.007 \\
GO:0055059 & Asymmetric neuroblast division & 1 & 0.014 \\
GO:0022008 & Neurogenesis & 9 & 0.014 \\
GO:0048812 & Neuron projection morphogenesis & 5 & 0.018 \\
GO:0031630 & Regulation of synaptic vesicle fusion to presynaptic & 1 & 0.018 \\
& active zone membrane & & 0.021 \\
GO:2000465 & Regulation of glycogen (starch) synthase activity & 1 & 0.024 \\
GO:0007399 & Nervous system development & 11 & 0.025 \\
GO:0007611 & Learning or memory & 3 & 0.027 \\
GO:2001223 & Negative regulation of neuron migration & 1 & 0.028 \\
GO:0048699 & Generation of neurons & 8 & 0.029 \\
GO:0031175 & Neuron projection development & 6 & 0.030 \\
GO:1990403 & Embryonic brain development & 1 & 0.032 \\
GO:0036445 & Neuronal stem cell division & 1 & 0.032 \\
GO:0055057 & Neuroblast division & 1 & 0.033 \\
GO:0007613 & Memory & 2 & 0.038 \\
GO:0007272 & Ensheathment of neurons & 2 & 0.039 \\
GO:0050890 & Cognition & 3 & 0.043 \\
GO:0016082 & Synaptic vesicle priming & 1 & 0.043 \\
GO:0030182 & Neuron differentiation & 7 &
\end{tabular}

GO, gene ontology; miR, microRNA; hsa, Homo sapiens. 
A

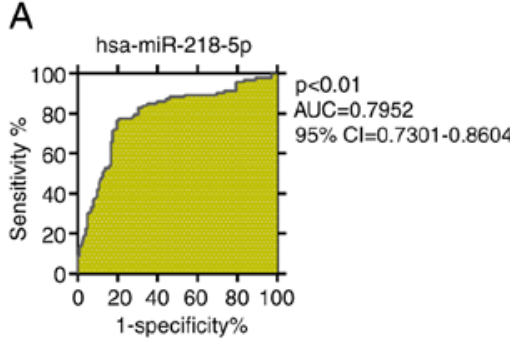

B

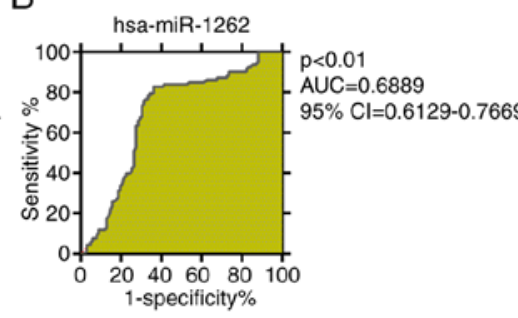

C

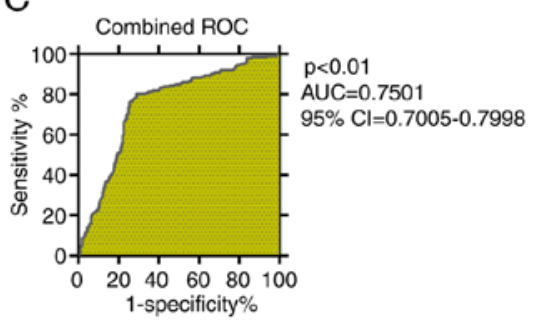

Figure 6. Diagnostic evaluation of 2 miRNAs. ROC curves of (A) hsa-miR-218-5P and (B) hsa-miR-1262, and (C) their combined ROC curve. ROC, receiver operating characteristic; AUC, area under the curve; miRNA/miR, microRNA; hsa, Homo sapiens.

the development of precise psychiatric medical treatments. The ENIGMA-Diffusion Tensor Imaging working group confirmed that the normal variation of human white matter is genetically associated with mental, neurological and developmental disorders $(44,45)$. The ENIGMA consortium performed a meta-analysis of 760 patients with SCZ and 957 healthy participants and revealed that the cognitive function of patients with SCZ is associated with the connectivity of the overall brain structure (46). Another study indicated that antipsychotic drugs resulted in an acute extrapyramidal response in the body by acting on two single nucleotide polymorphisms (SNPs; rs938112 and rs2987902) of two genes (LSMAP and ABL1) and destroying the binding sites of key transcription factors (47), LSAMP is an adhesion molecule of neuronal cells that participates in Neurogenesis, neurite outgrowth and plasticity (48), Abelson non-receptor tyrosine kinase (ABL1) is related to neurodegenerative diseases such as Parkinson's disease (48); the schizophrenia genomics platform is able to further elucidate the pathogenesis of SCZ (49) and provide therapeutic prospects for personalized therapy (50).

It is well known that the therapeutic effect of antipsychotic drugs is mainly exerted through the blocking of dopamine D2 receptor and/or the serotonin type $2 \mathrm{~A}$ receptor to reduce dopaminergic neurotransmission in the limbic midbrain. However, even though the molecular targets of psychotropic drugs have remained to be fully elucidated, several psychotropic drugs used in the clinic still comprise the cornerstone of psychosis treatment. Furthermore, clozapine is the only evidence-based treatment for TRS $(51,52)$, with $60-70 \%$ of patients reporting relief of their symptoms following this treatment (53); however, at present, there are no evidence-based pharmacotherapies for the remaining $30 \%$ of TRS patients who are not responsive to clozapine (54). Therefore, it is important to maximize the effectiveness of the drug through personalized treatment (55). The aim of pharmacogenomics (PGx) is to study how genes control the pharmacokinetics and pharmacodynamics of drugs (56). The concept may be used interchangeably with pharmacogenetics: A specific gene or group of genes may affect the patient's response to drugs. Ongoing randomized controlled trials have evaluated the clinical utility of PGx testing on antipsychotics in patients with SCZ to probe the association between genes and drugs on SCZ (trial registration nos. NCT02573168 and NCT02566057). Furthermore, the feasibility of genome-wide association studies, determination of polygenic risk scores and epigenetics of DNA methylation may render PGx more suitable for clinical research (57).
Based on the above concepts of precision medicine and PGx, even the application of epigenetics in SCZ research (58), combined with the fact that TRS is a highly heritable mental disorder and its heritability is markedly stronger than that of drug-responsive SCZ (59) the aim of the present study was to explore more sensitive treatment targets for TRS. Multiple studies have indicated that changes in cells and molecules in the brain may be relatively reflected by changes in the transcription in PBMCs (60-62), which may possess conjunct miRNA expression patterns with the brain (63). A previous study suggested that overexpressed miR-223 directly acts on mRNAs in plasma by the method of RT-qPCR and luciferase assay, revealing that it may be involved in the pathogenesis of SCZ (64). miR-30e, miR-181b, miR-34a, miR-346 and miR-7 in plasma may be useful non-invasive biomarkers for the diagnosis of SCZ, miR-132, miR-181b, miR-30e and miR-432 in plasma may be the improvement of symptoms in patients with SCZ, revealing potential indicators of their prognosis (65). Therefore, we can speculate that the abnormal expression levels of certain miRNAs in plasma may directly reflect the gene expression in the brain. Whether the expression of miRNA in PBMC is indirectly related to the expression of genes in the brain and the permeability of the blood-brain barrier of miRNAs need to be further studied. Fortunately, certain studies have confirmed the value of abnormally expressed miRNAs in the peripheral blood as biomarkers for SCZ $(66,67)$. Therefore, this novel, simple and simultaneously much cheaper strategy was explored by miRNA sequencing data analysis in the MCs derived from the peripheral blood of patients with TRS. With the assistance of bioinformatics analysis and RT-qPCR for validation, two biomarkers, hsa-miR-1262 and hsa-miR-218-5p, were first identified for the diagnosis of TRS, which exhibited significant alterations in patients with TRS, as compared with healthy controls. As there are no reports of these 2 miRNAs being associated with SCZ or other psychiatric diseases, hsa-miR-218-5p and hsa-miR-1262 underwent target gene predictions to provide further insight into the function of these two miRNAs using not only the TargetScan and Diana-microT online databases, but also GO and KEGG pathway analysis along with bioinformatics methods. The functions of these two miRNAs were indicated to be closely associated with brain development and function, including processes such as nervous system development, neuronal generation, neurogenesis and synapse function, and they were also mainly involved in dopaminergic synapses, Hippo signaling pathway-multiple species and GABAergic synapses, as demonstrated by KEGG analysis. Of note, certain 
studies on TRS candidate genes have focused on the dopaminergic and serotoninergic systems (68-72). SCZ is a complex disease that may be of neurodevelopmental origin and is characterized by abnormal synaptic plasticity at the cortical and subcortical levels, abnormal regulation of neurotransmitters (including dopamine, glutamate and serotonin) and abnormal signaling (73-75). Therefore, the ROC curve analysis reflected improved ability to differentiate patients with TRS from healthy controls (AUC value, 0.7501; 95\% CI, 0.7005-0.7998; specificity, 0.801; sensitivity, 0.711) in the present study, hsa-miR-218-5p and hsa-miR-1262, which have a critical role in the pathophysiological changes of TRS, were indicated to be promising sensitive biomarkers for TRS diagnosis, therapy and prediction.

The miRNA-gene network analysis of the present study indicated that there were three genes, namely, RNF165, CBX5, and CACUL1 targeted by the 2 miRNAs. Among these three genes, the identification of ring finger protein RNF165 was in accordance with previous studies, in which it was identified as a good candidate for SCZ diagnosis. In the study by Pickard et al (76), five key genes, including TTMA, TCF4, DLGAP1, ARKL1 and ARKL2, were confirmed as biomarkers for SCZ diagnosis. Along with the development of SCZ gene diagnosis, more biomarkers were gradually identified, including CBX5 (also termed HP1), which serves as a platform for other repressive proteins, such as DNA methyltransferases, and has been indicated to have a role in the functioning of dopamine neurons and the construction of memory as a member of heterochromatin in the brain. In addition, recently identified CACUL1, also known as CAC1 and C10orf46, is a novel gene of the cullin family that was reported to promote cell proliferation in a gastric cancer cell line (77), serves as a potential antitumor target in prostate cancer (78), promotes the proliferation of human lung cancer (79) and has an important role in protecting cells from $\mathrm{A} \beta$ toxicity and oxidative stress in the hippocampus of patients with Alzheimer's disease (80). The Pharmacogenomics Knowledgebase (PharmGKB) may provide candidate antipsychotic drug target genes for research (81). A drug-gene interaction study indicated that the rs 6313 position of the serotonin receptor gene HTR2A has a regulatory mechanism involving risperidone and olanzapine in SCZ (82). Another study indicated that gene variation of GABRB1 may be significantly associated with the antipsychotic drug dose (83). A strong connection has been reported between genetic polymorphisms (in the HSPG2, CNR1, DPP6 and SLC18A2 genes) and antipsychotic drugs, and even the side effects of these drugs (84). Another study identified 12 SNPs from 143 genes of 79 Han Chinese patients with SCZ via HaloPlex technology, with $2 / 12$ SNPs determined to be significantly associated with the response to olanzapine, using the MassARRAY platform in two independent cohorts (85). Other studies confirmed that the effectiveness of antipsychotic drugs is closely associated with the variation of drug target genes in patients with TRS (86-89). Although these three genes (RNF165, CBX5 and CACUL1) have not been reported in the PharmGKB to interact with medicines, the above information may be useful for future drug research.

In conclusion, in the present study, a simple, fast and effective strategy, mainly based on the detection of the expression of the top three miRNAs in peripheral blood MCs, was applied. hsa-miR-218-5p and hsa-miR-1262 were proposed as two major biomarkers for earlier and more precise diagnosis of TRS, shedding new light on the development of both gene-based diagnosis and treatment. However, the present study had certain limitations. First, the sample size of the study was small. Furthermore, limitations regarding the diagnostic accuracy may lead to misdiagnosis of certain samples. For example, the AUC value in ROC analysis was not very high, and there may be false positives and false negatives. Another limitation of this study is that the inclusion criteria were not designed to tell apart patients with normal schizophrenia that may respond to treatment from those with TRS. Finally, although the two target genes were confirmed in a relatively large cohort, there was a lack of functional verification, such as gene knockout experiments. In the future, studies with larger samples and a multi-center design are required to further verify the present results.

\section{Acknowledgements}

Not applicable.

\section{Funding}

The current study was supported by The National Natural Science Fund (grant no. 81760253).

\section{Availability of data and materials}

The datasets used and/or analyzed in this study are available from the corresponding author on reasonable request.

\section{Authors' contributions}

YZ and ZWT designed and supervised the study. XY, YQZ and QL were responsible for analyzing the data, writing the manuscript and revising it, and designing the figures. ZJL, $\mathrm{XM}, \mathrm{ZXL}, \mathrm{WY}, \mathrm{ZF}$ and WYZ contributed to the statistical analysis of the data and text correction. All authors read and approved the final version.

\section{Ethics approval and consent to participate}

Not applicable.

\section{Patient consent for publication}

Not applicable.

\section{Competing interests}

The authors declare that they have no competing interests.

\section{References}

1. Kahn RS, Sommer IE, Murray RM, Meyer-Lindenberg A, Weinberger DR, Cannon TD, O'Donovan M, Correll CU, Kane JM, van Os J and Insel TR: Schizophrenia. Nat Rev Dis Primers 1: 15067, 2015. 
2. Gillespie AL, Samanaite R, Mill J, Egerton A and MacCabe JH Is treatment-resistant schizophrenia categorically distinct from treatment-responsive schizophrenia? A systematic review. BMC Psychiatry 17: 12, 2017.

3. Lehman AF, Lieberman JA, Dixon LB, McGlashan TH, Miller AL, Perkins DO and Kreyenbuhl J; American Psychiatric Association; Steering Committee on Practice Guidelines: Practice guideline for the treatment of patients with schizophrenia, second edition. Am J Psychiatry 161: 1-56, 2004.

4. Samara MT, Dold M, Gianatsi M, Nikolakopoulou A, Helfer B, Salanti G and Leucht S: Efficacy, acceptability, and tolerability of antipsychotics in treatment-resistant schizophrenia: A network meta-analysis. JAMA Psychiatry 73: 199-210, 2016.

5. Lakhan SE and Kramer A: Schizophrenia genomics and proteomics: Are we any closer to biomarker discovery? Behav Brain Funct 5: 2, 2009.

6. Schwarz E and Bahn S: Biomarker discovery in psychiatric disorders. Electrophoresis 29: 2884-2890, 2008

7. Schwarz E and Bahn S: The utility of biomarker discovery approaches for the detection of disease mechanisms in psychiatric disorders. Br J Pharmacol 153: S133-S136, 2008.

8. Qureshi IA and Mehler MF: Emerging roles of non-coding RNAs in brain evolution, development, plasticity and disease. Nat Rev Neurosci 13: 528-541, 2012.

9. Tsankova N, Renthal W, Kumar A and Nestler EJ: Epigenetic regulation in psychiatric disorders. Nat Rev Neurosci 8: 355-367, 2007.

10. Sullivan PF, Fan C and Perou CM: Evaluating the comparability of gene expression in blood and brain. Am J Med Genet B Neuropsychiatr Genet 141: 261-268, 2006.

11. Vawter MP, Ferran E, Galke B, Cooper K, Bunney WE and Byerley W: Microarray screening of lymphocyte gene expression differences in a multiplex schizophrenia pedigree. Schizophr Res 67: 41-52, 2004.

12. Glatt SJ, Everall IP, Kremen WS, Corbeil J, Sásik R, Khanlou N, Han M, Liew CC and Tsuang MT: Comparative gene expression analysis of blood and brain provides concurrent validation of SELENBP1 up-regulation in schizophrenia. Proc Natl Acad Sci USA 102: 15533-15538, 2005.

13. Kuzman MR, Medved V, Terzic J and Krainc D: Genome-wide expression analysis of peripheral blood identifies candidate biomarkers for schizophrenia. J Psychiatr Res 43: 1073-1077, 2009.

14. Maffioletti E, Tardito D, Gennarelli M and Bocchio-Chiavetto L: Micro spies from the brain to the periphery: New clues from studies on microRNAs in neuropsychiatric disorders. Front Cell Neurosci 8 $75,2014$.

15. Yan Y, Chen X, Wang X, Zhao Z, Hu W, Zeng S, Wei J, Yang X, Qian L, Zhou S, et al: The effects and the mechanisms of autophagy on the cancer-associated fibroblasts in cancer. J Exp Clin Cancer Res 38: 171, 2019

16. Li H, Zhou DS, Chang H, Wang L, Liu W, Dai SX, Zhang C, Cai J, Liu W, Li X, et al: Interactome Analyses implicated CAMK2A in the genetic predisposition and pharmacological mechanism of bipolar disorder. J Psychiatr Res 115: 165-175, 2019.

17. Yan Y, Gong Z and Xu Z: Commentary: Lico A causes ER stress and apoptosis via up-regulating miR-144-3p in human lung cancer cell line H292. Biomed J 41: 391-392, 2018.

18. Ambros V: The functions of animal microRNAs. Nature 431: 350-355, 2004.

19. Xu Z, Yan Y, Zeng S, Dai S, Chen X, Wei J and Gong Z: Circular RNAs: Clinical relevance in cancer. Oncotarget 9: 1444-1460, 2018

20. Perkins DO, Jeffries C and Sullivan P: Expanding the "central dogma: The regulatory role of nonprotein coding genes and implications for the genetic liability to schizophrenia. Mol Psychiatry 10 $69-78,2005$

21. Cheng HY, Papp JW, Varlamova O, Dziema H, Russell B, Curfman JP, Nakazawa T, Shimizu K, Okamura H, Impey S and Obrietan K: MicroRNA modulation of circadian-clock period and entrainment. Neuron 54: 813-829, 2007.

22. Kocerha J, Faghihi MA, Lopez-Toledano MA, Huang J, Ramsey AJ, Caron MG, Sales N, Willoughby D, Elmen J, Hansen HF, et al: MicroRNA-219 modulates NMDA receptor-mediated neurobehavioral dysfunction. Proc Natl Acad Sci USA 106: 3507-3512, 2009.

23. Coyle JT: MicroRNAs suggest a new mechanism for altered brain gene expression in schizophrenia. Proc Natl Acad Sci USA 106 2975-2976, 2009.

24. Miller BH and Wahlestedt C: MicroRNA dysregulation in psychiatric disease. Brain Res 1338: 89-99, 2010.

25. Gladkevich A, Kauffman HF and Korf J: Lymphocytes as a neural probe: Potential for studying psychiatric disorders. Prog Neuropsychopharmacol Biol Psychiatry 28: 559-576, 2004.
26. Marques-Deak A, Cizza G and Sternberg E: Brain-Immune interactions and disease susceptibility. Mol Psychiatry 10: 239-250, 2005.

27. Marazziti D, Catena Dell'osso M, Baroni S, Masala I, Dell'Osso B, Consoli G, Giannaccini G, Betti L and Lucacchini A: Alterations of the dopamine transporter in resting lymphocytes of patients with different psychotic disorders. Psychiatry Res 175: 54-57, 2010

28. Lai CY, Yu SL, Hsieh MH, Chen $\mathrm{CH}$, Chen HY, Wen CC, Huang YH, Hsiao PC, Hsiao CK, Liu CM, et al: MicroRNA expression aberration as potential peripheral blood biomarkers for schizophrenia. PLoS One 6: e21635, 2011.

29. Sun Xy, Lu J, Zhang L, Song Ht, Zhao L, Fan Hm, Zhong Af, Niu W, Guo Zm, Dai Yh, et al: Aberrant microRNA expression in peripheral plasma and mononuclear cells as specific blood-based biomarkers in schizophrenia patients. J Clin Neurosci 22: 570-574, 2015.

30. Wei H, Yuan Y, Liu S, Wang C, Yang F, Lu Z, Wang C, Deng $\mathrm{H}$, Zhao J, Shen $\mathrm{Y}$, et al: Detection of circulating miRNA levels in schizophrenia. Am J Psychiatry 172: 1141-1147, 2015.

31. Liu S, Zhang F, Shugart YY, Yang L, Li X, Liu Z, Sun N, Yang C, Guo X, Shi J, et al: The early growth response protein 1-miR-30a-5p-neurogenic differentiation factor 1 axis as a novel biomarker for schizophrenia diagnosis and treatment monitoring. Transl Psychiatry 7: e998, 2017.

32. Kalia M and Silva JC: Biomarkers of psychiatric diseases: Current status and future prospects. Metabolism 64: S11-S15, 2015.

33. Tandon R, Gaebel W, Barch DM, Bustillo J, Gur RE, Heckers S, Malaspina D, Owen MJ, Schultz S, Tsuang M, et al: Definition and description of schizophrenia in the DSM-5. Schizophr Res 150: 3-10, 2013

34. Rao X, Huang X, Zhou Z and Lin X: An improvement of the $2^{\wedge}$ (-delta delta CT) method for quantitative real-time polymerase chain reaction data analysis. Biostat Bioinforma Biomath 3: 71-85, 2013.

35. Agarwal V, Bell GW, Nam JW and Bartel DP: Predicting effective microRNA target sites in mammalian mRNAs. ELife 4: e05005, 2015.

36. Paraskevopoulou MD, Georgakilas G, Kostoulas N, Vlachos IS, Vergoulis T, Reczko M, Filippidis C, Dalamagas T and Hatzigeorgiou AG: DIANA-microT web server v5.0: Service integration into miRNA functional analysis workflows. Nucleic Acids Res 41: W169-W173, 2013.

37. Wang X, Su R, Guo Q, Liu J, Ruan B and Wang G: Competing endogenous RNA (ceRNA) hypothetic model based on comprehensive analysis of long non-coding RNA expression in lung adenocarcinoma. Peer J 7: e8024, 2019.

38. Meltzer HY: Treatment-resistant schizophrenia-the role of clozapine. Curr Med Res Opin 14: 1-20, 1997.

39. Wimberley T, Gasse C, Meier SM, Agerbo E, MacCabe JH and Horsdal HT: Polygenic risk score for schizophrenia and treatment-resistant schizophrenia. Schizophr Bull 43: 1064-1069, 2017.

40. Ashley EA: Towards precision medicine. Nat Rev Genet 17: 507-522, 2016

41. Tonelli MR and Shirts BH: Knowledge for precision medicine: Mechanistic reasoning and methodological pluralism. JAMA 318: 1649-1650, 2017.

42. Alda M: Personalized psychiatry: Many questions, fewer answers. J Psychiatry Neurosci 38: 363-365, 2013.

43. Manchia M, Pisanu C, Squassina A and Carpiniello B: Challenges and future prospects of precision medicine in psychiatry. Pharmgenomics Pers Med 13: 127-140, 2020.

44. Kochunov P, Hong LE, Dennis EL, Morey RA, Tate DF, Wilde EA, Logue M, Kelly S, Donohoe G, Favre P, et al: ENIGMA-DTI: Translating reproducible white matter deficits into personalized vulnerability metrics in cross-diagnostic psychiatric research. Human Brain Mapp 16: 24998, 2020.

45. Thompson PM, Jahanshad N, Ching CR, Salminen LE, Thomopoulos SI, Bright J, Baune BT, Bertolín S, Bralten J, Bruin WB, et al: ENIGMA and global neuroscience: A decade of large-scale studies of the brain in health and disease across more than 40 countries. Transl Psychiatry 10: 100, 2020.

46. Holleran L, Kelly S, Alloza C, Agartz I, Andreassen OA, Arango C, Banaj N, Calhoun V, Cannon D, Carr V, et al: The relationship between white matter microstructure and general cognitive ability in patients with schizophrenia and healthy participants in the ENIGMA consortium. Am J Psychiatry 177: 537-547, 2020. 
47. Boloc D, Rodríguez N, Torres T, García-Cerro S, Parellada M Saiz-Ruiz J, Cuesta MJ, Bernardo M, Gassó P, Lafuente A, et al: Identifying key transcription factors for pharmacogenetic studies of antipsychotics induced extrapyramidal symptoms. Psychopharmacology (Berl) 237: 2151-2159, 2020.

48. Heinla I, Leidmaa E, Kongi K, Pennert A, Innos J, Nurk K, Tekko T, Singh K, Vanaveski T, Reimets R, et al: Gene expression patterns and environmental enrichment-induced effects in the hippocampi of mice suggest importance of lsamp in plasticity. Front Neurosci 9: 205,2015 .

49. Rees E and Owen MJ: Translating insights from neuropsychiatric genetics and genomics for precision psychiatry. Genome Med 12: 43, 2020.

50. Birnbaum R and Weinberger DR: Special article: Translational science update. Pharmacological implications of emerging schizophrenia genetics: Can the bridge from 'Genomics' to 'Therapeutics' be defined and traversed? J Clin Psychopharmacol 40: 323-329, 2020.

51. Chakos M, Lieberman J, Hoffman E, Bradford D and Sheitman B Effectiveness of second-generation antipsychotics in patients with treatment-resistant schizophrenia: A review and meta-analysis of randomized trials. Am J Psychiatry 158: 518-526, 2001.

52. Wahlbeck K, Cheine M, Essali A and Adams C: Evidence of clozapine's effectiveness in schizophrenia: A systematic review and meta-analysis of randomized trials. Am J Psychiatry 156: 990-999, 1999.

53. Meltzer HY: Treatment of the neuroleptic-nonresponsive schizophrenic patient. Schizophr Bull 18: 515-542, 1992.

54. Siskind DJ, Lee M, Ravindran A, Zhang Q, Ma E, Motamarri B and Kisely S: Augmentation strategies for clozapine refractory schizophrenia: A systematic review and meta-analysis. Aust N Z J Psychiatry 52: 751-767, 2018

55. van Westrhenen R, Aitchison KJ, Ingelman-Sundberg $M$ and Jukić MM: Pharmacogenomics of antidepressant and antipsychotic treatment: How far have we got and where are we going? Front Psychiatry 11: 94, 2020.

56. Relling MV and Evans WE: Pharmacogenomics in the clinic. Nature 526: 343-350, 2015

57. Zhang JP and Malhotra AK: Recent progress in pharmacogenomics of antipsychotic drug response. Curr Psychiatry Rep 20: 24, 2018.

58. Feinberg AP and Fallin MD: Epigenetics at the crossroads of genes and the environment. JAMA 314: 1129-1130, 2015.

59. Nucifora FC, Woznica E, Lee BJ, Cascella N and Sawa A: Treatment resistant schizophrenia: Clinical, biological, and therapeutic perspectives. Neurobiol Dis 131: 104257, 2019.

60. Fisar Z and Raboch J: Depression, antidepressants, and peripheral blood components. Neuro Endocrinol Lett 29: 17-28, 2008.

61. Cattaneo A, Sesta A, Calabrese F, Nielsen G, Riva MA and Gennarelli M: The expression of VGF is reduced in leukocytes of depressed patients and it is restored by effective antidepressant treatment. Neuropsychopharmacology 35: 1423-1428, 2010

62. Chen SD, Sun XY, Niu W, Kong LM, He MJ, Fan HM, Li WS Zhong AF, Zhang LY and Lu J: A preliminary analysis of microRNA-21 expression alteration after antipsychotic treatment in patients with schizophrenia. Psychiatry Res 244: 324-332, 2016.

63. Liang Y, Ridzon D, Wong L and Chen C: Characterization of microRNA expression profiles in normal human tissues. BMC Genomics 8: 166, 2007.

64. Zhao Z, Jinde S, Koike S, Tada M, Satomura Y, Yoshikawa A, Nishimura Y, Takizawa R, Kinoshita A, Sakakibara E, et al: Altered expression of microRNA-223 in the plasma of patients with first-episode schizophrenia and its possible relation to neuronal migration-related genes. Transl Psychiatry 9: 289, 2019.

65. Sun Xy, Zhang J, Niu W, Guo W, Song Ht, Li Hy, Fan Hm, Zhao L, Zhong Af, Dai Yh, et al: A preliminary analysis of microRNA as potential clinical biomarker for schizophrenia. Am J Med Genet B Neuropsychiatr Genet 168: 170-178, 2015.

66. He K, Guo C, He L and Shi Y: miRNAs of peripheral blood as the biomarker of schizophrenia. Hereditas 155: 9, 2018

67. Liu S, Zhang F, Wang X, Shugart YY, Zhao Y, Li X, Liu Z, Sun N, Yang C, Zhang K, et al: Diagnostic value of blood-derived microRNAs for schizophrenia: Results of a meta-analysis and validation. Sci Rep 7: 15328, 2017.

68. Inada T, Nakamura A and Iijima Y: Relationship between catechol-O-methyltransferase polymorphism and treatment-resistant schizophrenia. Am J Med Genet B Neuropsychiatr Genet 120: $35-39,2003$

69. Ji X, Takahashi N, Saito S, Ishihara R, Maeno N, Inada T and Ozaki N: Relationship between three serotonin receptor subtypes (HTR3A, HTR2A and HTR4) and treatment-resistant schizophrenia in the Japanese population. Neurosci Lett 435: 95-98, 2008.
70. Kohlrausch FB, Gama CS, Lobato MI, Belmonte-de-Abreu P, Callegari-Jacques SM, Gesteira A, Barros F, Carracedo A and Hutz MH: Naturalistic pharmacogenetic study of treatment resistance to typical neuroleptics in European-Brazilian schizophrenics. Pharmacogenet Genomics 18: 599-609, 2008.

71. Bilic P, Jukic V, Vilibic M, Savic A and Bozina N: TreatmentResistant schizophrenia and DAT and SERT polymorphisms. Gene 543: 125-132, 2014.

72. Terzić T, Kastelic M, Dolžan V and Plesničar BK: Influence of 5-HT1A and 5-HTTLPR genetic variants on the schizophrenia symptoms and occurrence of treatment-resistant schizophrenia. Neuropsychiatr Dis Treat 11: 453-459, 2015.

73. Catts VS, Fung SJ, Long LE, Joshi D, Vercammen A, Allen KM, Fillman SG, Rothmond DA, Sinclair D, Tiwari Y, et al: Rethinking schizophrenia in the context of normal neurodevelopment. Front Cell Neurosci 7: 60, 2013.

74. Alexander-Bloch AF, Reiss PT, Rapoport J, McAdams H, Giedd JN, Bullmore ET and Gogtay N: Abnormal cortical growth in schizophrenia targets normative modules of synchronized development. Biol Psychiatry 76: 438-446, 2014.

75. Horváth S and Mirnics K: Schizophrenia as a disorder of molecular pathways. Biol Psychiatry 77: 22-28, 2015.

76. Pickard BS, Malloy MP, Clark L, Lehellard S, Ewald HL, Mors O, Porteous DJ, Blackwood DH and Muir WJ: Candidate psychiatric illness genes identified in patients with pericentric inversions of chromosome 18. Psychiatr Genet 15: 37-44, 2005.

77. Zheng Q, Zhao LY, Kong Y, Nan KJ, Yao Y and Liao ZJ: CDK-Associated cullin 1 can promote cell proliferation and inhibit cisplatin-induced apoptosis in the AGS gastric cancer cell line. World J Surg Oncol 11: 5, 2013.

78. Choi H, Lee SH, Um SJ and Kim EJ: CACUL1 functions as a negative regulator of androgen receptor in prostate cancer cells. Cancer Lett 376: 360-366, 2016.

79. Chen TJ, Gao F, Yang T, Thakur A, Ren H, Li Y, Zhang S, Wang T and Chen MW: CDK-Associated cullin 1 promotes cell proliferation with activation of ERK1/2 in human lung cancer A549 cells. Biochem Biophys Res Commun 437: 108-113, 2013.

80. Kong Y, Bai Ps, Sun H and Nan Kj: Expression of the newly identified gene $\mathrm{CACl}$ in the hippocampus of alzheimer's disease patients. J Mol Neurosci 47: 207-218, 2012.

81. Drögemöller BI, Wright GE, Niehaus DJ Emsley R and Warnich L: Next-generation sequencing of pharmacogenes: A critical analysis focusing on schizophrenia treatment. Pharmacogenet Genomics 23: 666-674, 2013.

82. Maffioletti E, Valsecchi P, Minelli A, Magri C, Bonvicini C, Barlati S, Sacchetti E, Vita A and Gennarelli M: Association study between HTR2A rs6313 polymorphism and early response to risperidone and olanzapine in schizophrenia patients. Drug Dev Res 27: doi:10, 2020.

83. Hettige NC, de Morae GH, Kennedy JL and De Luca V: Candidate gene analysis of pharmacodynamic targets for antipsychotic dosage. Pharmacogenomics 17: 199-208, 2016.

84. Zai CC, Tiwari AK, Zai GC, Maes MS and Kennedy JL: New findings in pharmacogenetics of schizophrenia. Curr Opin Psychiatry 31: 200-212, 2018.

85. Zhou W, Xu Y, Lv Q, Sheng YH, Chen L, Li M, Shen L, Huai C, Yi Z, Cui D and Qin S: Genetic association of olanzapine treatment response in Han Chinese schizophrenia patients. Front Pharmacol 10: 177, 2019.

86. Ruderfer DM, Charney AW, Readhead B, Kidd BA, Kähler AK, Kenny PJ, Keiser MJ, Moran JL, Hultman CM, Scott SA, et al: Polygenic overlap between schizophrenia risk and antipsychotic response: A genomic medicine approach. Lancet Psychiatry 3: 350-357, 2016

87. Gressier F, Porcelli S, Calati R and Serretti A: Pharmacogenetics of clozapine response and induced weight gain: A comprehensive review and meta-analysis. Eur Neuropsychopharmacol 26: 163-185, 2016.

88. Zhang JP and Malhotra AK: Pharmacogenetics and antipsychotics: Therapeutic efficacy and side effects prediction. Exp Opin Drug Metab Toxicol 7: 9-37, 2011.

89. Sriretnakumar V, Huang E and Müller DJ: Pharmacogenetics of clozapine treatment response and side-effects in schizophrenia: An update. Exp Opin Drug Metab Toxicol 11: 1709-1731, 2015.

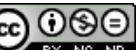

This work is licensed under a Creative Commons

Attribution-NonCommercial-NoDerivatives 4.0 International (CC BY-NC-ND 4.0) License. 